\title{
Particle Identification and Tracking by the Use of a Pixel- Based Semiconductor Radiation Detector Coupled with Voltage Controlled Oscillators
}

\author{
K. Coulié ${ }^{1}$, W. Rahajandraibe ${ }^{1}$, L. Ottaviani ${ }^{1}$ \\ ${ }^{1}$ Aix Marseille Université, CNRS, Université de Toulon, IM2NP UMR 7334, Marseille, France \\ Corresponding author: karine.coulie@im2np.fr
}

\begin{abstract}
A particle detection chain based on a CMOSSOI VCO circuit associated to a matrix of detection is presented. The solution is optimized for the recognition and tracking of various particles. Two ions are considered: an alpha and an aluminum. These two ions were chosen because there are very different in terms of energy and LET variations.
\end{abstract}

Keywords - Sensor, detection, VCO, radiation.

\section{INTRODUCTION}

S EMICONDUCTOR radiation detectors, also called solid-state detectors, can be classified into several categories, depending on the application field and the particles to be detected. These detectors are based on charge collection and amplification using a semiconductor volume such as a p-n junction [1-2]. They exhibit several advantages in comparison with other types of detectors such as gas-filled counters. For instance, they usually offer faster chargecollection time which provide the ability to process higher counting rates. Another advantage is their compactness which allows the measurement of intensity variations over small distances, as well as their low electron-hole pair energy threshold, improving the measurement resolution. A pixel organization including several sensors could be used when a high detector sensitivity is required [2-8]. This configuration will be investigated in this work.

Particles counting and/or recognition require the conditioning of the signal generated by the sensor. Most of the existing conditioning chains are based on a direct reading of the detected currents created by the crossing of an ionizing particle through the matter. When an embedded reading system is necessary, this solution could not be suitable for the detection of low energy particles where the current signature could be a narrow pulse with a duration of few nanoseconds. Indeed, the readout chain must ensure sufficiently high resolution (optimized Signal-to-Noise-Ratio) for an appropriate postprocessing. Moreover, a reading amplifier exhibiting a fast response time is required which means a higher current consumption and complex circuit implementation. Designing high precision, lowpower wideband amplifier is a critical issue. As a consequence, most practical detectors are based on direct reading of the detected currents using Charge Sensitive Amplifiers (CSA) [9]. In this case, the circuit delivers a limited amount of information on the actual current shape and no information about the electrical signature of the particles is available. In applications such as gas prospection or medical therapy, the characteristics of the electrical signature of the particles must be known. Moreover, the knowledge of the generated current shape at the output of the detector allows easier post processing of the signal.

In this context, a new approach based on the use of a ring oscillator was developed [10]. This approach is based on the reading of the information related to indirect output parameters of the detection chain signal (i.e. voltage variation), instead of directly measuring the current from the sensor. This solution avoids most of the design problems described upper. Then, the information is extracted by correlating the initial oscillating signal of the system with the oscillating signal after the particle has passed the detector. The particle recognition only requires to link the output information (i.e. voltage variation of the oscillating signal) to the input information (current stimuli). The detection system presented here should be enough sensitive to detect low energy particle, to track ions and to provide information about the shape of the detected currents. The 


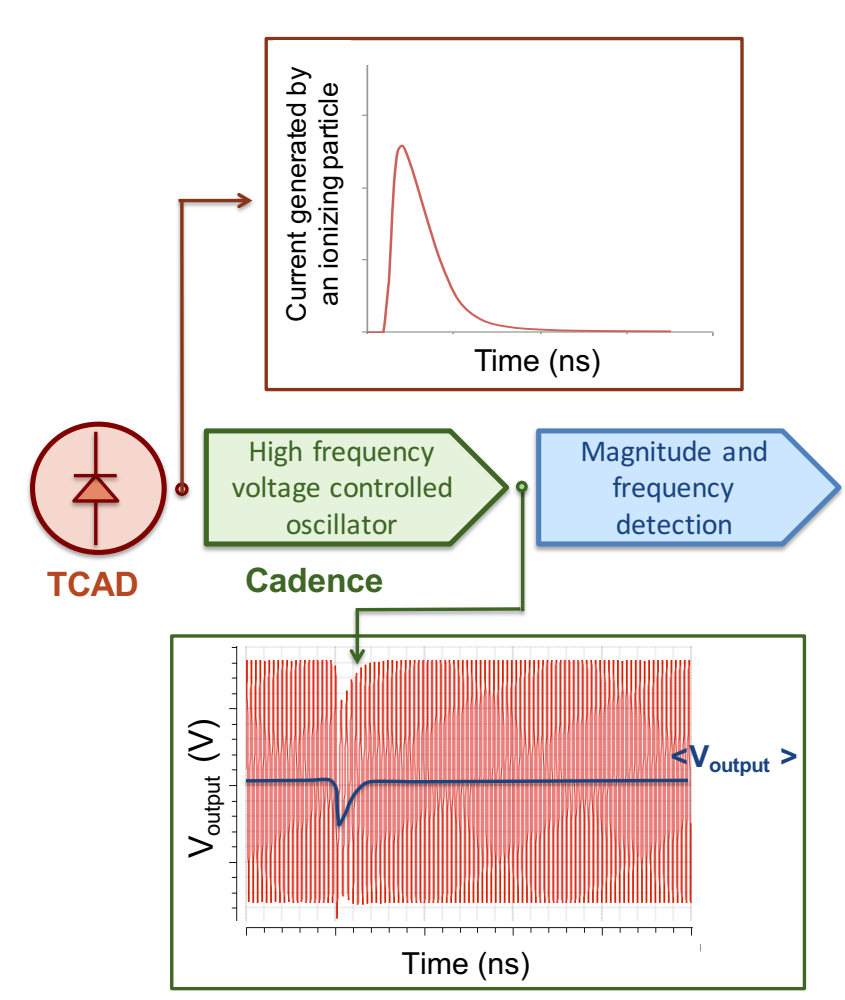

Fig. 1. Simulation chain including the current from TCAD modeling (top) and the variation of the output voltage versus time at the output of the VCO (bottom).

analyses are performed using the device simulator of Synopsys ${ }^{\circledR}$ CAO tool for current generation and the electrical simulator of Cadence ${ }^{\circledR}$ for the VCO simulation.

The full detection system (VCO + matrix) will be described in section II. Section III is dedicated to the system characterization which is mandatory for particle recognition. Part IV deals with two case studies: an alpha particle and an aluminum ion and section $\mathrm{V}$ presents a complementary method to allow charge determination. The last section concludes the paper.

\section{THE DETECTION SYSTEM}

\section{A. The VCO chain}

The detection chain has been designed and implemented on $130 \mathrm{~nm}$ CMOS SOI technology, then simulated at circuit level using "Spectre" simulator (SPICE-based) under Cadence Virtuoso (C) $\mathrm{CAO}$ tool. The concept itself was presented in [1013]. The VCO chain is composed of three parts: a CMOS based pixel detector, a shaping circuit based on a Voltage Controlled ring Oscillator (VCO) and a system for frequency and magnitude detection (Fig.
1). This system allows the evaluation of the circuit sensitivity to radiation by measuring the oscillator responses. In the very first versions of the system, the operating frequency of $0,35 \mu \mathrm{m}$ Bulk Silicon substrate CMOS VCO was $1 \mathrm{MHz}$. Thanks to a new solution based on another CMOS process $(130 \mathrm{~nm}$ $\mathrm{SOI}$ ), it reaches now $4.3 \mathrm{GHz}$. This increase of the operating frequency makes possible the development of new methods for signal recognition. As actual operating frequencies are reaching $4.3 \mathrm{GHz}$, the shape of the signal is directly reproduced at the output of the VCO. Then, the output parameter is now the average voltage variation (Fig.1). The advantage of this solution is a direct recognition of the signal shape as an amplitude modulation of the VCO output frequency.

\section{B. The detection matrix}

In order to improve the efficiency of the detection and the tracking ability, a $3 \times 5$ matrix is modeled and analyzed (Fig. 2). The input current comes from the realistic simulation of the matrix using TCAD device simulation tools (Synopsis (C)). The effect of the ion strike is simulated using the Heavy Ion module of Synopsys [14], considering an electron-hole pair column centered on the ion track axis. The Linear Energy Transfer is defined as the energy lost by the

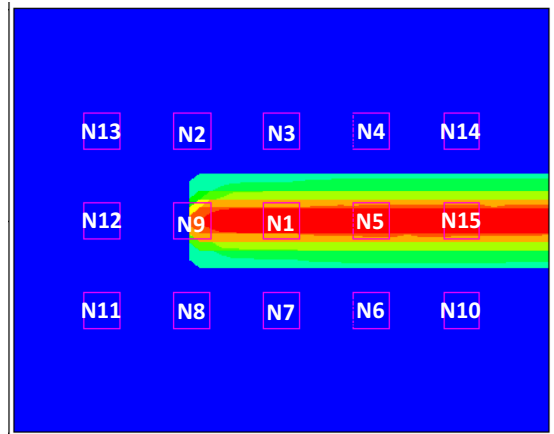

Fig. 2. Top view of the fifteen contacts matrix for the simulation of the alpha particle.

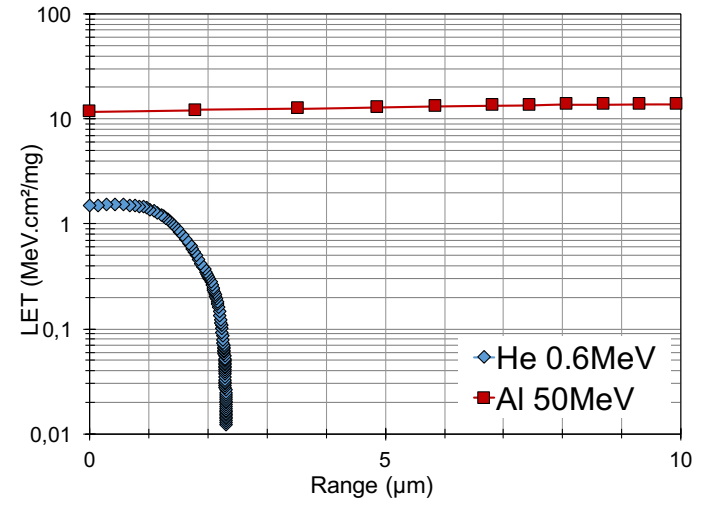

Fig. 3. LET versus range for the aluminum and alpha ions. 


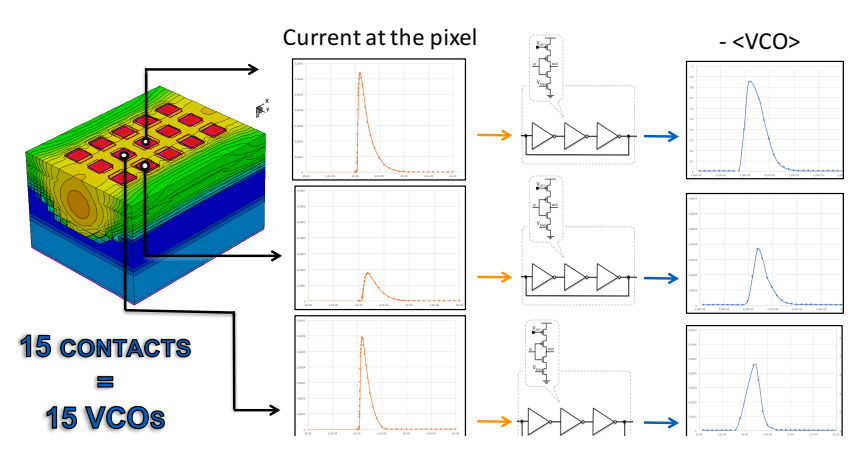

Fig. 4. Schematic presenting the process of simulation for three pixels.

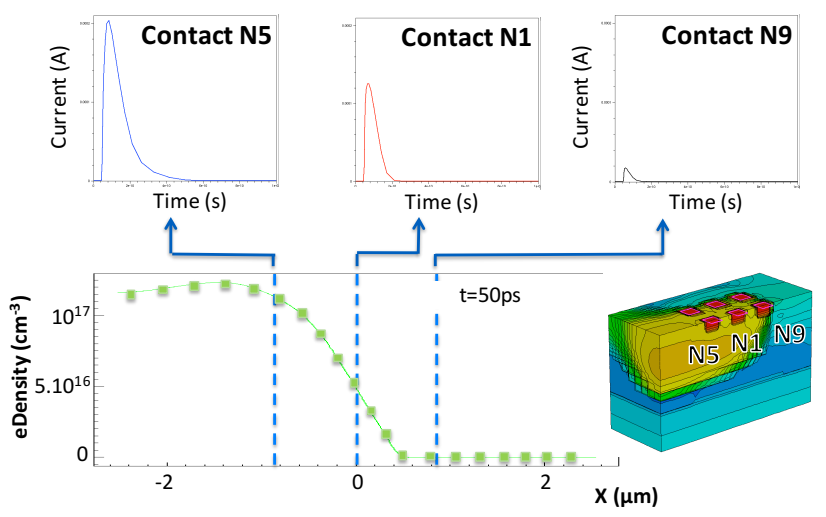

Fig. 5. Example of variation of the current at the output of three pixels related to the electron density, for an alpha particle generated horizontally.

particle, by unit of length and varies along the track depending on the initial energy of the particles [15]. An actual variation of the LET was integrated in our simulations, based on the value given by SRIM tables [16] (Fig. 3).

As this detector should be suitable for low energy particles detection, one of the simulated ion is an alpha particle crossing the device with an initial energy of $0.6 \mathrm{MeV}$, which corresponds to a maximum range of $2.3 \mu \mathrm{m}$ in the silicon matrix. The aim is to study the end of the path corresponding to an alpha particle of $1.47 \mathrm{MeV}$ generated by the initial interaction of thermal neutron with boron-10. The case of a more energetic particle is also considered. This is a $50 \mathrm{MeV}$ aluminum ion which could be produced by the interaction of fast protons with silicon [17]. The maximum of the charge generation occurs at $50 \mathrm{ps}$.

\section{The whole system}

Thus, the effect of the chosen particle in the detection cell is simulated at device level and the oscillator at circuit level. The current generated at each electrode of the matrix is injected at the corresponding oscillator input. The average voltage is taken from the output oscillating signal of the VCO. Then if the matrix includes 15 contacts, 15 oscillators are needed (Fig. 4).

\section{THE SYSTEM CHARACTERIZATION}

\section{A. Information for tracking}

Several information can be provided by the currents at the pixels. First one, is the charge density variation. The electron density variation is plotted versus the particle path on Fig. 5. At the top of this figure, the currents extracted from the contacts just above the charge generation are presented. The variation of the currents clearly follows the one of the charge density. The highest current, on the left, corresponds to the highest electron density and the lowest current, on the right, corresponds to the lowest charge density. These results, coming from the simulation of a $3 \times 3$ matrix presented in [19], show that the current variation can provide an information about the charge density in the detection cell.

In [19], it was also shown that the current shape can also give information about the distance between the charge generation and the contact. Indeed, for a contact located just above the ion, the current peak will appear earlier than for contacts located further from the generation point.

Another important result is that the average voltage from the VCO clearly fits the current variation from the pixels. This is clearly visible in the example of

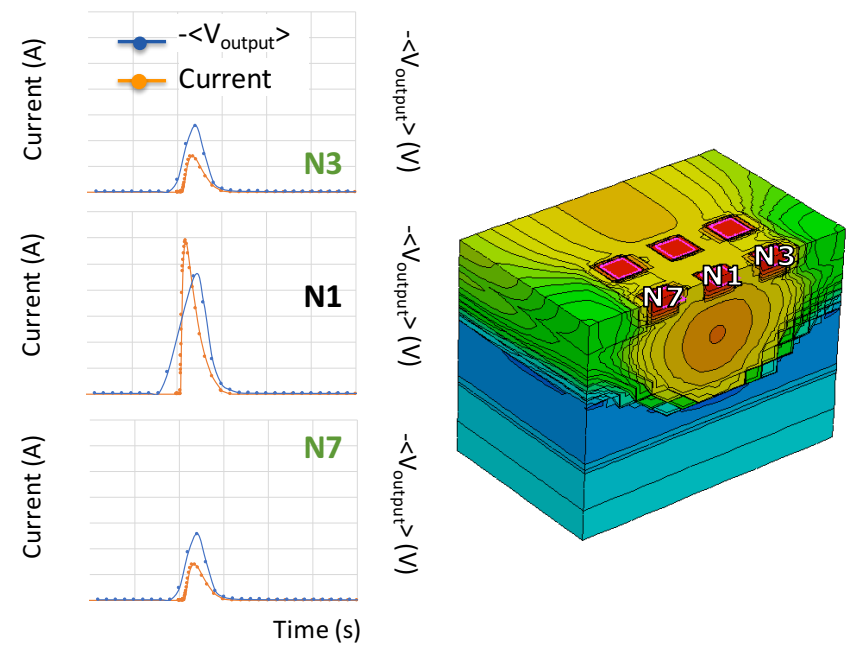

Fig. 6. Example of variation of the average output voltage of the VCO for three pixels related to the input current from the matrix, for an alpha particle generated horizontally. 


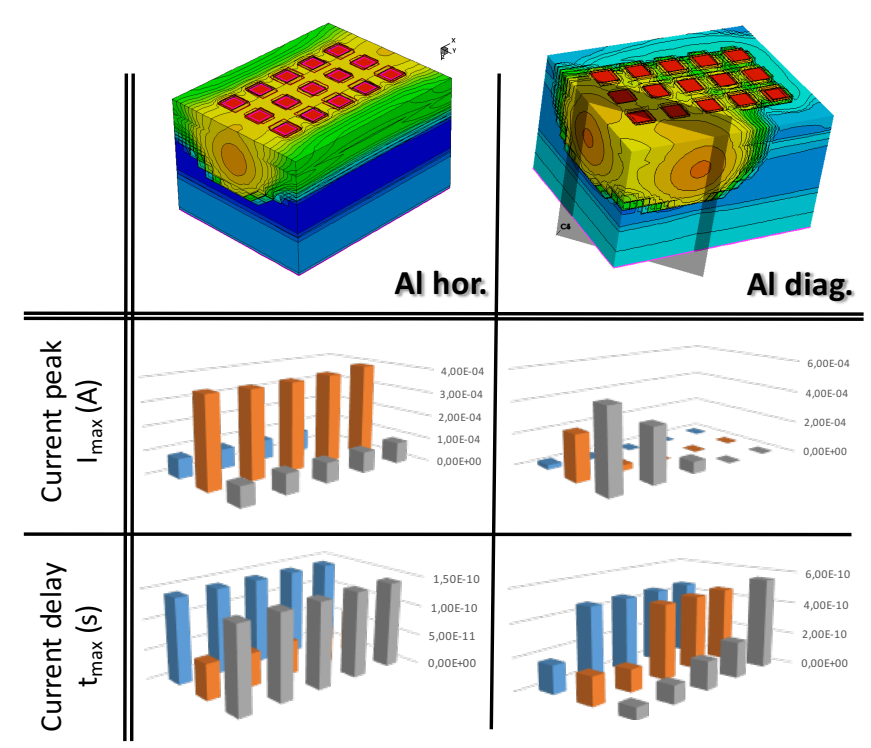

Fig. 7. Variation of the current peak and current delay at the output of the 15 pixels for the aluminum generated horizontally and diagonally.

Fig. 6. This corresponds to the horizontal strike of an alpha particle presented in Fig.5 [19]. The cut is in the direction perpendicular to the particle path. The orange curves correspond to the current from the pixel and the blue curves to the average voltage from the VCO. The scale is the same for the 3 presented contacts. The symmetry of the track can be seen by comparing the green contacts corresponding to top and bottom graphs. Their currents are clearly the same. The current corresponding to the black contact is higher because it is located just above the ion.

\section{B. Calibration of the VCO chain}

The key point is how the output parameters of the VCO chain can give information related to the input current. This could be done through the analysis of various characteristics extracted from the average voltage variation. In a previous work, we demonstrated that several metrics can be used but we decide to mainly focus on the variation of the average output signal $(\Delta V \max )$ versus the maximum of the input current (Imax). In [19], a linearity curve linking input to output parameters was determined for the 4.3 $\mathrm{GHz}$ VCO studied here. The curve which characterizes the VCO response is named « calibration curve ». It is obtained by the injection of various analytical currents at the input of the VCO. As this curve is linear, through calibration curves, the output parameters can be linked to the input currents, which could allow the incoming particle identification.

\section{CASES STUdy}

\section{A. Ion tracking}

\section{1) Case of an aluminum ion}

The first case study deals with the crossing of an aluminum ion in the 15 pixels structure. On the left of Fig. 7, the horizontal case is presented and on the right, the diagonal case. We check here the opportunity to perform particle tracking from the currents from the detection matrix. Two metrics are explored: the current peak and the time corresponding to this current peak. We know that these two parameters can give information about the charge density and the position of the particle strike. For both presented cases, the particle impact is visible on the current peak and the current delay. Indeed, the current peak is clearly higher for the contacts just above the particle and decreases for the most distant contacts. Concerning the current delay, it is lower for the contacts just above and increases for the most distant contacts.

The horizontal configuration has been extensively studied in [19] so we will focus on the diagonal configuration. Fig. 8 presents the electron density at depth $=0.8 \mu \mathrm{m}$. The local electron density is presented in Fig. 9 for $\mathrm{t}=50 \mathrm{ps}$, which corresponds to the maximum time of the generation.

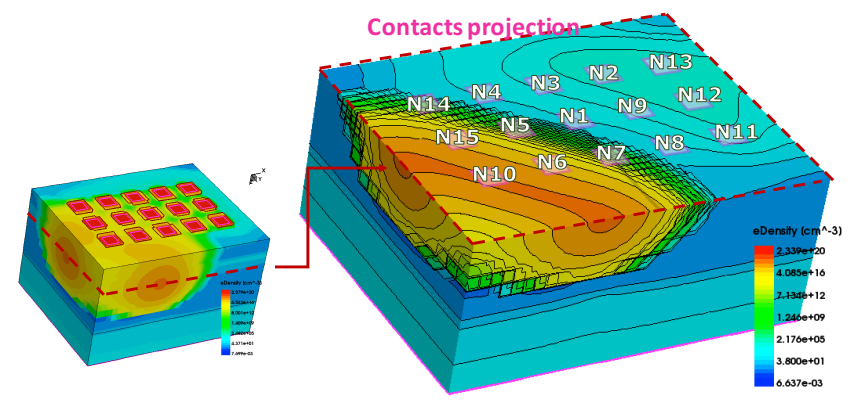

Fig. 8. Aluminum generation in the 15-pixels matrix. The figure on the right is a cut at depth $=0.8 \mu \mathrm{m}$. The represented contacts are a projection of the fifteen top contacts.

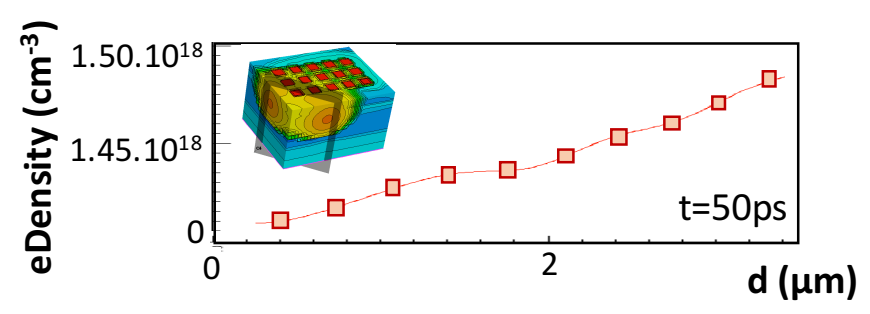

Fig. 9. Electron density along the particle track for the aluminum particle. 


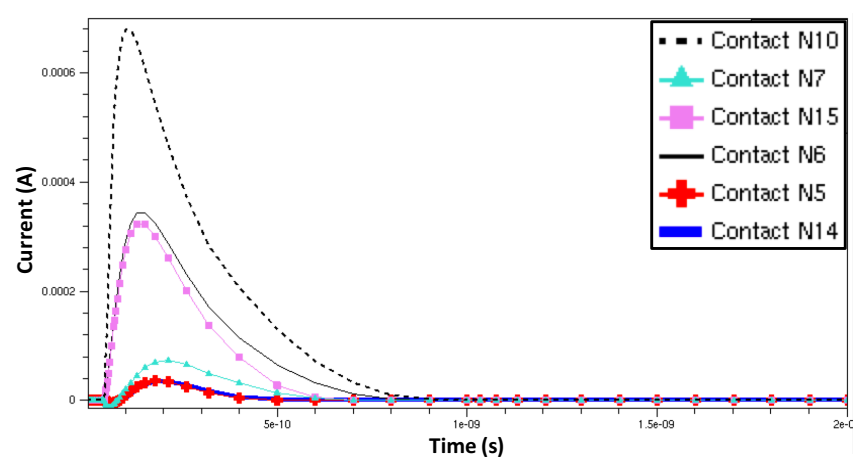

Fig. 10. Comparison of the currents of the six highest currents (N10, N15, $\mathrm{N} 6, \mathrm{~N} 7, \mathrm{~N} 5$ and N14 contacts) for the $5 \times 3$ matrix, aluminum particle.
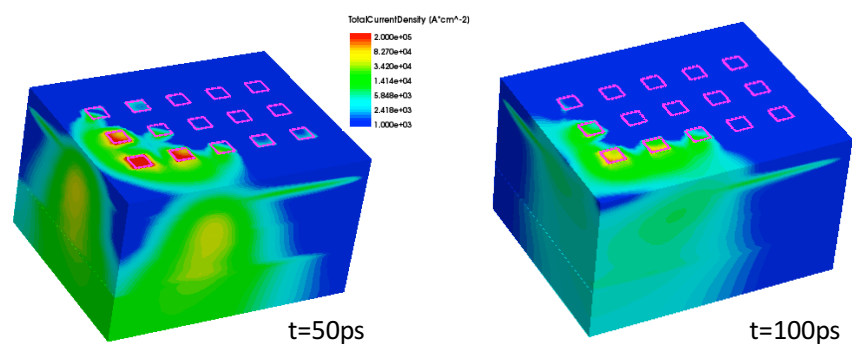

Fig. 11. Total current density at $\mathrm{t}=50 \mathrm{ps}$ (maximum of the generation) and $\mathrm{t}=100 \mathrm{ps}$ for the aluminum particle.

The six highest currents collected during the particle crossing are shown in Fig. 10. The highest current corresponds to N10 because this contact is just under the particle track. The maximum of the generation appears at $\mathrm{t}=108 \mathrm{ps}$. Two other currents correspond to the N6 and N15 contacts. The maximum time corresponding to these currents is the same: $t=138$ ps. The N6 current is higher because the local electron density increases along the track (Fig. 9). The N14 and N5 currents are the same and seem to be at the same distance from the track. However, a highest current would have been expected for N5 because the electron density increases along the track. It could be the effect of the surrounding contacts as it has been highlighted in [19]. The N7 current is higher and its maximum generation time arrives later than for N5 and N14. The N7 contact is probably further from the track. The total current density is shown in Fig. 11. The same variation is observed and visible even at $\mathrm{t}=100$ ps.

The total collected charge for this configuration is $400 \mathrm{fC}$. This gives an information about the deposited energy.

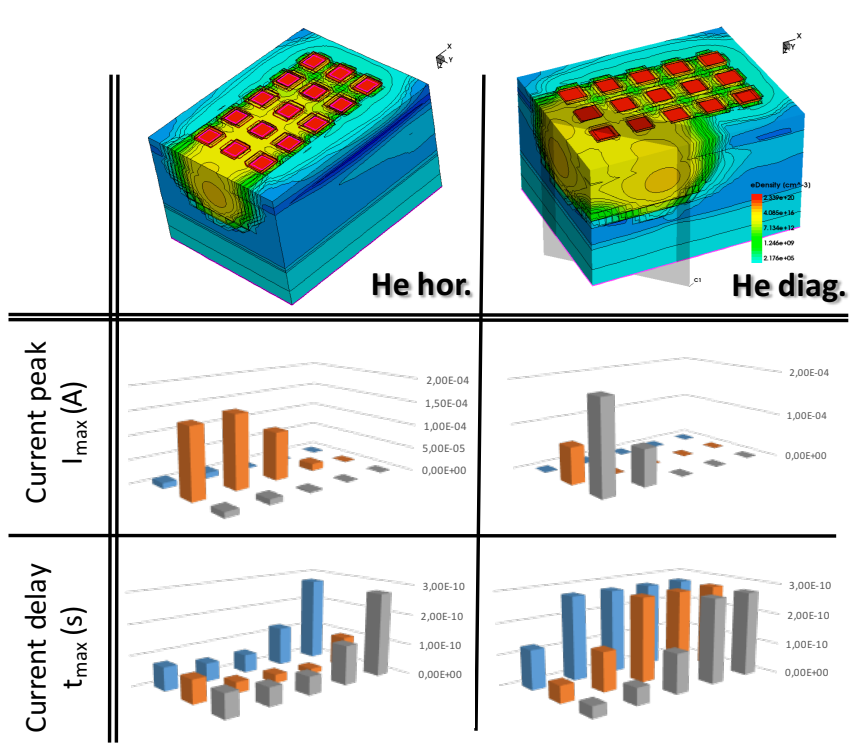

Fig. 12. Variation of the current peak and current delay at the output of the 15 pixels for the alpha generated horizontally and diagonally.

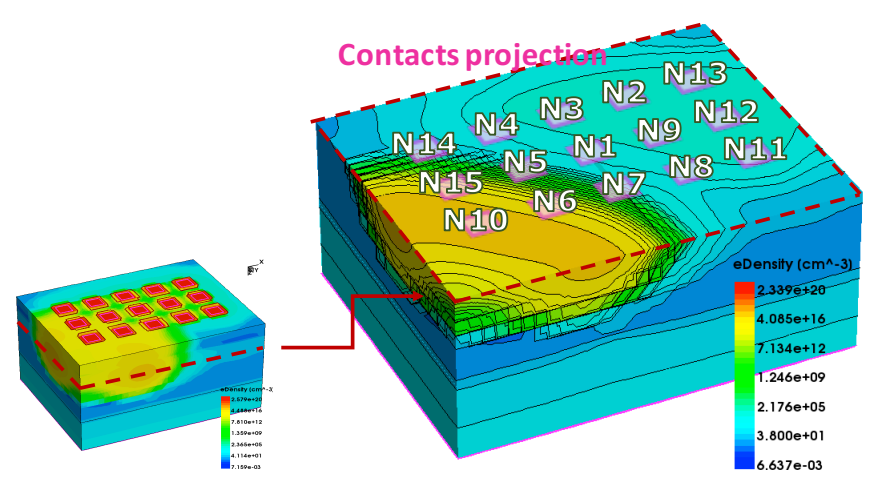

Fig. 13. Alpha generation in the 15-pixels matrix. The figure on the right is a cut at depth $=0.8 \mu \mathrm{m}$. The represented contacts are a projection of the fifteen top contacts.

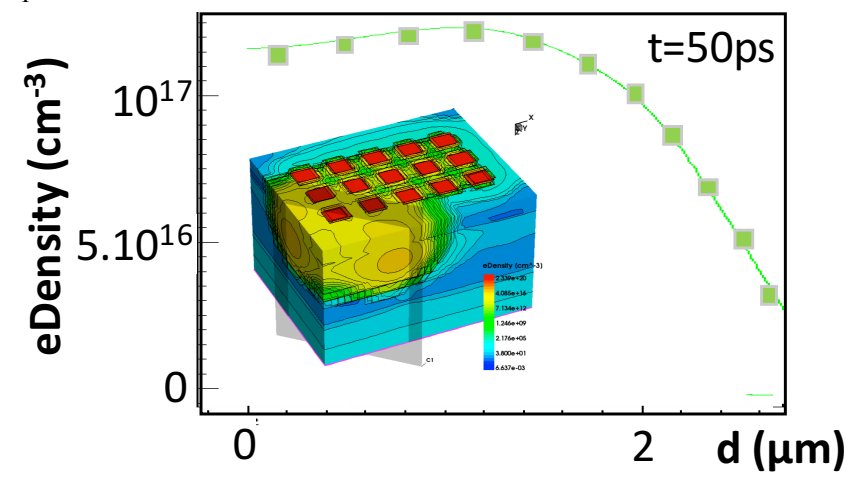

Fig. 14. Electron density along the particle track for the alpha particle.

\section{2) Case of an alpha particle}

The second case study deals with the crossing of an alpha particle in the same structure. Once again, the current peak and the current delay follow the expected trend and the particle tracking is possible. As for the aluminum particle, Fig. 13 presents the 


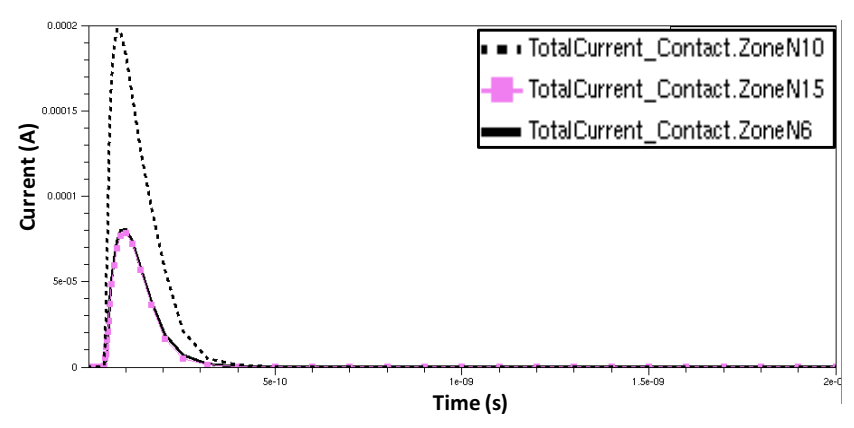

Fig. 15. $5 \times 3$ matrix (15 contacts). Comparison of the currents of the three highest currents (N10, N15, N6 contacts) for the alpha particle.

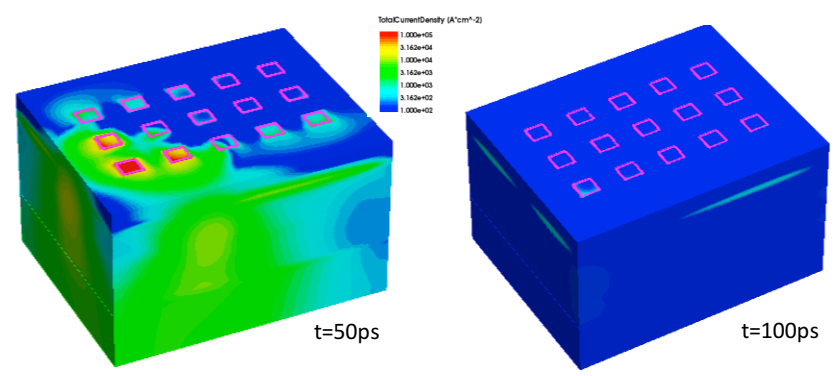

Fig. 16. Total current density at $\mathrm{t}=50 \mathrm{ps}$ (maximum of the generation) and $\mathrm{t}=100 \mathrm{ps}$ for the alpha particle.

electron density at depth $=0.8 \mu \mathrm{m}$. The local electron density is presented in Fig. 14 for $t=50$ ps, which corresponds to the maximum time of the generation.

The three highest currents collected during the particle crossing are shown in Fig. 15. The highest one corresponds to N10 contact. This contact is just under the particle track. The maximum of the generation appears at $\mathrm{t}=78 \mathrm{ps}$. The two other currents correspond to the N6 and N15 contacts. They are approximatively at the same distance from the trace. This is visible through the maximum generation time which is the same for both contacts: 96 ps. As the electron density follows a bell curve, it is almost the same at both contacts. The total current density is shown in Fig. 16. We can state that the same variation is observed. By analyzing the maximum current and the maximum generation time, the particle crossing can be approximately determined. The maximum generation time gives an idea of the distance between the ion and the contact. Then thanks to Fig. 15, we know that the N10 contact is closer from the particle than the N6 and N15 contacts.

The total collected charge for this configuration is $42,8 \mathrm{fC}$ which is about 10 times lower than for the aluminum.

\section{3) VCO detection}

As previously said, the possibility to detect alpha and aluminum horizontal tracks has been checked in a previous study. So we will focus on diagonal configurations. Plotting the corresponding output versus input response, we can see that all the currents are detected, the trend is preserved and even for the shortest currents, the detection is possible (Fig. 17). Only one point on the graph is out of the $50 \%$ detection zone what is an unexpected behavior. So we had a look on the average voltages.

All the currents corresponding to both particles in diagonal configuration have been injected sequentially in the VCO. The average voltage is presented in Fig. 18 for the highest currents corresponding to the two particles. All the currents of Fig. 10 and 15 are correctly reproduced except

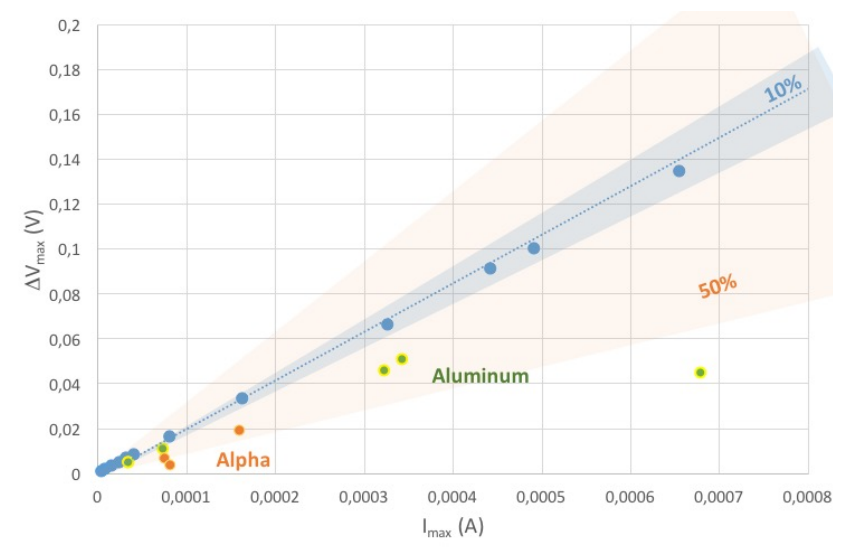

Fig. 17. Calibration curve and detected currents concerning diagonal configurations for the aluminum ion or the alpha particle crossing in a fifteen contacts matrix.

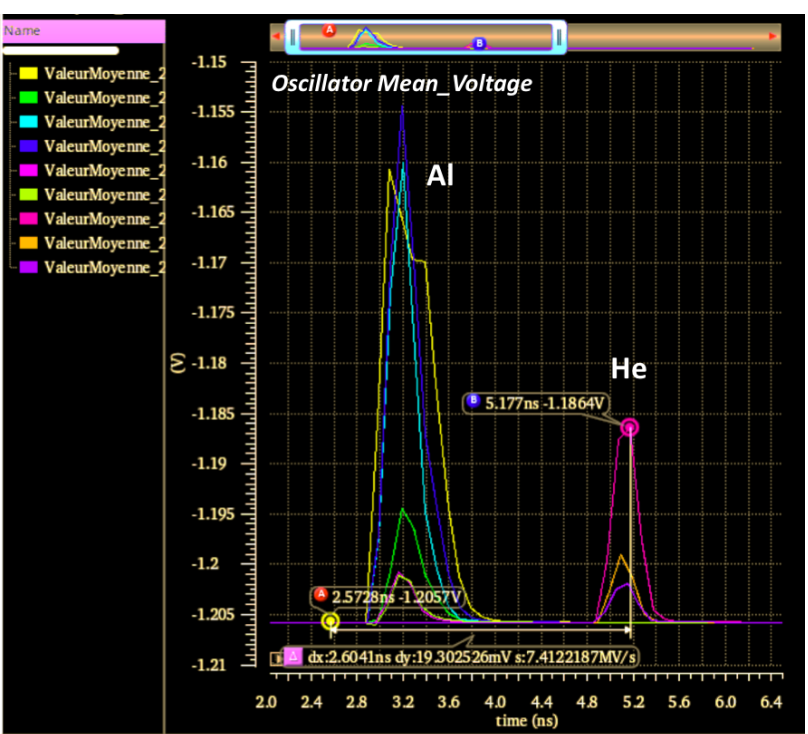

Fig. 18. Mean voltage for the highest currents: aluminum on the left, alpha on the right. 


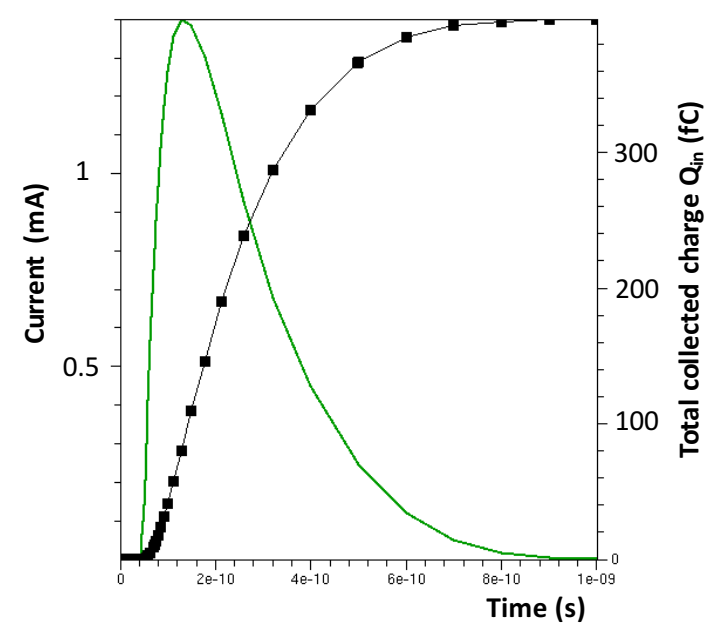

Fig. 19. Current at the bottom contact of the pixel (green curve) and corresponding total collected (black curve) versus time.

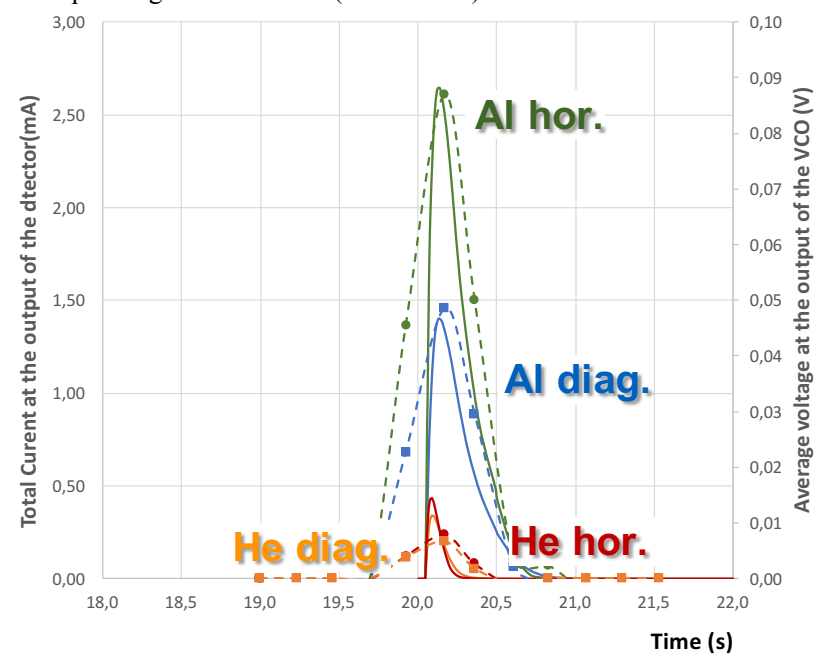

Fig. 20. Current at the bottom contact of the pixel (full line curves) and corresponding average voltage (dotted line curves) versus time.

the N10 current of the aluminum, visible on Fig. 10 and corresponding to the yellow average voltage of Fig. 18. This is surprising because this current is large enough to be correctly detected by the VCO. This current is too high and the oscillator saturates. It is not mandatory for the particle tracking but important for the determination of the total collected charge. Then we decide to use another oscillator, with a lower operating frequency for charge detection. This oscillator is not suitable for particle tracking because it is too slow. But it is able to drive a higher current at the input.

\section{Determination OF THE TOTAL COLlected CHARGE}

The addition of all the currents collected at the fifteen pixels of the matrix corresponds to the current collected at the bottom electrode of the matrix. An example of this bottom current is presented on Fig. 19. The total collected charge, in black, corresponds to the integral of this current. This bottom current lasts about $1 \mathrm{~ns}$ what is large enough to be detected by the $2.1 \mathrm{GHz}$ VCO we have selected for charge detection. This VCO was presented in a previous paper [18].

Then we injected the 4 bottom currents, corresponding to our 4 configurations (He hor., He diag., Al hor., Al diag.), at the input of a $2.1 \mathrm{GHz}$ oscillator. The current peaks are much higher than the one which saturated the $4.3 \mathrm{GHz}$ VCO (Fig. 20). Dotted curves correspond to the average voltages at the output of the VCO. The variation of these voltages follows the variation of the input currents and the $2.1 \mathrm{GHz}$ oscillator does not saturate, as required.

The linearity curve corresponding to the $2.1 \mathrm{GHz}$ oscillator was presented in a previous paper [18] (Fig. 21). The output charge corresponds to the integral of the average voltage. It is plotted versus the input charge corresponding to the integral of the current. The charges for aluminum and alpha particles are added on this graph. They are in the $20 \%$ detection zone which is a quite good result to allow particle identification.

Finally, the full detection system would require one oscillator oscillating at $2.1 \mathrm{GHz}$ for charge identification and $15 \mathrm{VCO}$ oscillating at $4.3 \mathrm{GHz}$ for particle tracking.

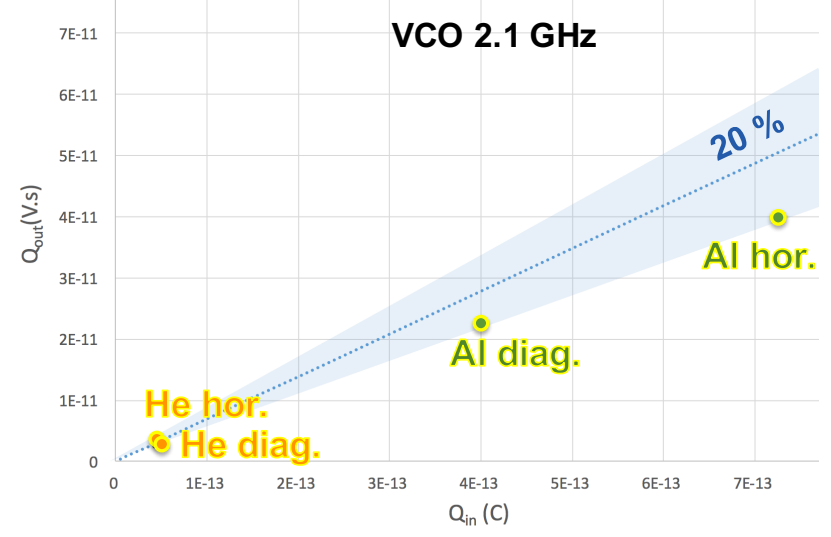

Fig. 21. Calibration curve for injected charge versus output charge, concerning diagonal configurations, for the aluminum ion or the alpha particle crossing in a fifteen contacts matrix. Only bottom currents are injected in the $2.1 \mathrm{GHz} \mathrm{VCO}$. 


\section{CONCLUSIONS}

Finally, with a VCO oscillating at $4.3 \mathrm{GHz}$ we demonstrate that tracking various kinds of particles is possible. It is also possible to get information about the total collected charge by using a $2.1 \mathrm{GHz} \mathrm{VCO}$.

Now, we would like to optimize the detector response in order to improve the detection efficiency, especially by exploring the possibility to use a faster oscillator for charge identification. We are also working on the material implementation of the voltage controlled oscillator and its associated electronics.

\section{REFERENCES}

[1] C. Fiorini, A. Pullia, E. Gatti, A. Longoni, and W. Buttler, "A monolitic implementation of a switch-current 'wheel' amplifier for multi-channel silicon drift detectors, IEEE Trans. Nucl. Sci., vol. 46 n³ p. 161, 1999.

[2] D. Hussona, A. Bozierb, S. Higueretc, T.D. Lec, A. Nourreddinea, "AlphaRad, a new integrated CMOS System-on-Chip for high efficiency alpha particles counting", Nuclear Instruments and Methods in Physics Research A 569., pp. 845-852 161, 2006.

[3] I. Perić, C. Kreidl, P. Fischer, "Particle pixel detectors in high-voltage CMOS technology-New achievements", Nuclear Instruments and Methods in Physics Research A, Vol. 650, Issue 1, p. 158-162, 2011.

[4] M. Trocmé, S. Higueret, D. Husson, A. Nourreddine, T.D. Lê, "A new compact device for efficient neutron counting using a CMOS active pixel sensor", Radiation Measurements, pp. 1100-1103, 2008.

[5] D. Passeri, P. Placidi, L. Verducci, P. Ciampolini, G. Matrella, A. Marras, G/M. Bilei, "High-resolution CMOS particle detectors: design and test issues", 331-334 Vol.1, 2003 IEEE Nuclear Science Symposium Conference Record.

[6] M. Campbell, V. Havranek, E. HEIjne, T. Holy, J. Idarraga, J. Jakubek, C. Lebel, C. Leray, X. Llopart, J. Novotny, S. Pospisil, L. Tlustos, Z. Vykydal, "Charge collection from proton and alpha particle tracks in silicon pixel detector devices”, 2007 IEEE Nuclear Science Symposium Conference Record.

[7] M. J. Baudot ; G. Bertolone ; A. Brogna ; G. Claus ; C. Colledani et al., "First test results Of MIMOSA-26, a fast CMOS sensor with integrated zero suppression and digitized output", 2009 IEEE Nuclear Science Symposium Conference Record, 2009.

[8] Tomas Baca ; Martin Jilek ; Petr Manek ; Petr Stibinger ; Vladimir Linhart ; Jan Jakubek ; Martin Saska, “Timepix Radiation Detector for Autonomous Radiation Localization and Mapping by Micro Unmanned Vehicles”, 2019 IEEE/RSJ International Conference on Intelligent Robots and Systems (IROS), 3-8 Nov. 2019.

[9] Ying Zhang, Christine Hu-Guo, Daniel Husson, The-Duc Le, Stéphane Higueret, Yann Hu, "A High-Sensitivity Low-Power CMOS Sensor for a Future Neutron Personal Dosimeter", in IEEE Trans. On Nucl. Sc., vol.59, no.4, pp. 1465-1471, August 2012.

[10] Rahajandraibe W., Aziza H., Coulié-Castellani K., Micolau, "Device and Method for Detecting Radiation Particles"

G. Patent AMU/CNRS/UAPV - Septembre 17, 2015 WO2015136220, https://patentscope.wipo.int/search/en/detail.jsf?docId=WO2015136220

[11] K. Castellani-Coulié, H. Aziza, W. Rahajandraibe, G. Micolau, J.-M. Portal, "Development of a CMOS Oscillator Concept for Particle Detection and Tracking", IEEE Trans Nucl. Sci., Vol. 60 , Issue 4 , pp. 2450 - 2455 , Aug. 2013. DOI: 10.1109/TNS.2013.2254723

[12] K. Coulié-Castellani, W. Rahajandraibe, G. Micolau, H. Aziza, J.-M. Portal, "Improvement of a detection chain based on a VCO concept for microelectronic reliability under natural radiative environment", IEEE LATW Conference, 2015.

[13] S. Ben Krit, K. Coulié-Castellani, W. Rahajandraibe, L. Ottaviani, G. Micolau, H. Aziza, J.-M. Portal, "Comparison of a Readout Chain Dedicated to the Signal Conditioning of a Particle Detector and an
Innovative Chain Based on a VCO Concept", RADECS 2015, Moscou, Russia, September 2015. DOI: 10.1109/RADECS.2015.7365592

[14] TCAD Sentaurus user's manual (Synopsys).

[15] A. Akkerman, J. Barak, D. Emfietzoglou, "Ion and electron trackstructure and its effects in silicon: model and calculation", Nuclear Instruments and Methods in Physics Research, pp. 319-336, 2005.

[16] www.srim.org

[17] Vasiliy V. Markelov and Michael G. Tverskoy, "Evaluation of LET Spectra Produced by High Energy Protons in Si", RADECS 2015, Moscou, Russia, September $2015 . \quad$ DOI: 10.1109/RADECS.2015.7365592

[18] K. Coulié, W. Rahajandraibe, H. Aziza, G. Micolau and R. Vauché, "Detection limit of a VCO based detection chain dedicated to particles recognition and tracking”, EPJ Web Conf., 170 (2018) 09002 , DOI: https://doi.org/10.1051/epjconf/201817009002

[19] K. Coulié, W. Rahajandraibe, H. Aziza, G. Micolau, R. Vauché, "Detection limit of a VCO based detection chain dedicated to particles recognition and tracking", 2005 8th European Conference on Radiation and Its Effects on Components and Systems, 2005. DOI: 10.1109/RADECS.2005.4365629. 